\title{
Does physician specialty matter?
}

\author{
Walter Montanera, MD, FRCP(C) \\ Department of Radiology, University of Toronto, St. Michael's Hospital, Toronto, Ontario, Canada
}

I $\mathrm{N}$ this paper, Fennell et al. ${ }^{1}$ examine the rates of complication and mortality for endovascular treatment of intracranial aneurysms (both ruptured and unruptured) as recorded in a database of US academic health centers. In their analysis, the rates vary significantly when evaluated against the subspecialty performing the endovascular procedure.

As stated by the authors, the limitations of the database are many, and the analysis is retrospective. Institutions and/or individuals "self-report" to the database and a "complication" is not defined or detailed. Is the reporting of complications homogenous among contributing institutions? Is a groin hematoma considered equal to an intraoperative aneurysm rupture or ischemic stroke? There are no data allowing comparison of the patients (such as age, comorbidities, subarachnoid hemorrhage grading, etc.), or aneurysms treated (such as size, location, neck width, etc.). Neurosurgeons would choose surgical clip placement for some patients, yet surgical morbidity and mortality is not included. Because we lack these details and comparisons, the potential for selection bias is significant.

As stated by the authors, there are no data concerning the procedure volumes for treating centers or individual practitioners. Higher volume centers may have a range of subspecialty expertise not available in lower volume centers, which could result in improved outcomes. It is possible that neurologists treat, on average, fewer patients than neurosurgeons. Therefore, apparent differences in morbidity and/or mortality could be related more to practitioner volume rather than specialty. Because the database upon which this analysis depends is far from complete, any conclusions regarding performance by specialty is, at best, unreliable and premature.

Fennell et al. further hypothesize that apparent lower morbidity and/or mortality rates among patients treated by neurosurgeons may be due to differences in pre-fel- lowship training and "more robust understanding of neurocritical care concepts." Virtually all neuroendovascular practitioners would agree that improved understanding and training in neurocritical care (as incorporated in neurosurgical training) would be of benefit and potentially improve patient outcomes. Similarly, better understanding of the detection and management of ischemic stroke (as incorporated in neurology training), or better analysis of CT/MRI/neuroangiographic details (as incorporated in neuroradiology training) may also improve patient outcomes. Any future analysis of morbidity and mortality in aneurysm treatment may choose to compare "collaborative" (multispecialty) management versus single specialty.

Fennell et al. conclude with a call for improved training and focus on patient outcomes, a statement with which few would disagree. Although their analysis does not provide an answer, their findings reiterate an important question: How do we optimize training for the neuroendovascular practitioner of the future? This analysis underscores the compelling need for reliable and robust data to aid accrediting organizations and professional associations in the future design and refinement of training programs in neuroendovascular management.

http://thejns.org/doi/abs/10.3171/2015.1.JNS142794

\section{Reference}

1. Fennell VS, Martirosyan NL, Palejwala SK, Lemole GM Jr, Dumont TM: Morbidity and mortality of patients with endovascularly treated intracerebral aneurysms: does physician specialty matter? J Neurosurg [epub ahead of print August 14, 2015. DOI: 10.3171/2014.11.JNS141030]

\section{Disclosure}

The author reports no conflict of interest. 


\section{Response}

Travis M. Dumont, MD, Vernard S. Fennell, MD, and G. Michael Lemole Jr., MD

Division of Neurosurgery, Department of Surgery, University of Arizona Medical Center, University of Arizona College of Medicine, Tucson, Arizona

We thank Dr. Montanera for his insightful commentary. He again highlights many of the limitations of the UHC Database that were used for this analysis. Specifically, in addition to missing individual case details, most physician data is censored and thus does not allow for comparison between practitioners, and in particular case volume, which is an obvious potential confounding factor that has been previously studied and reported as referenced.

The data set will, however, allow for comparison of center volume. To this end, we performed an additional analysis of the same data set to compare reported complication and mortality incidence based on hospital case volume. Our analysis was performed with the identical statistical methodology as in the original report. It is worth noting that the hospitals included in the UHC database are, by definition, university-based practices, which may trend toward higher volume, so this analysis carries its own limitations.
Defining "low volume" as being a case number below the median, and "high volume" as a case number above the median, we found similar rates of complications at lowand high-volume centers for both unruptured and ruptured aneurysms (Table 1). Mortalities were noted to be lower in the treatment of unruptured aneurysms at high-volume centers compared with low-volume centers, but mortality rates were similar in cases of aneurysmal rupture (Table 1). In this database during the studied time period, median reported case volume was 80 for unruptured and 54 for ruptured cases per hospital.

While this additional analysis does not eliminate the limitations of our paper, we hope it clarifies the point that while case volume is a factor, it does not take away from the primary conclusion of the report. We believe the primary conclusion of our paper is sound, and disagree with Dr. Montanera that the conclusions of the paper are "unreliable and premature." For the benefit of the reader, we repeat our primary conclusion: "In this study there was a statistically significant finding that neurosurgically trained physicians may demonstrate improved outcomes with respect to endovascular treatment of unruptured aneurysms in this cohort. This finding warrants further investigation." Indeed, we welcome higher-level evidence on this interesting topic.

TABLE 1. Reported incidence of complications and mortality for patients with treated aneurysms based on center volume

\begin{tabular}{llccc}
\hline Variable & Cases & Low-Volume Centers (\%) & High-Volume Centers (\%) & Chi-Square Value \\
\hline Complications & & & & 0.064 \\
\hline & Unruptured & 7.5 & 29.4 & 0.206 \\
\hline & Ruptured & 27.6 & & $<0.001$ \\
\hline Mortality & & 1.4 & 0.252 \\
\hline & Unruptured & 2.5 & 11.8 & \\
\hline
\end{tabular}

